\title{
Correction: Everolimus (EVE) and exemestane (EXE) in patients with advanced breast cancer aged $\geq 65$ years: new lessons for clinical practice from the EVA study \\ This article has been corrected. Correction in Oncotarget. 2018; 9:34639-34640.
}

Marina Cazzaniga ${ }^{1,2}$, Claudio Verusio ${ }^{3}$, Mariangela Ciccarese ${ }^{4}$, Alberto Fumagalli ${ }^{5}$, Donata Sartori ${ }^{6}$, Maria Rosaria Valerio ${ }^{7}$, Mario Airoldi ${ }^{8}$, Gabriella Moretti ${ }^{9}$, Corrado Ficorella ${ }^{10}$, Valentina Arcangelii ${ }^{11}$, Lucrezia Diodati ${ }^{12}$, Alberto Zambelli ${ }^{13}$, Antonio Febbraro $^{14}$, Daniele Generali ${ }^{15}$, Mirco Pistellii ${ }^{16}$, Ornella Garrone ${ }^{17}$, Antonino Musolino ${ }^{18}$, Patrizia Vici ${ }^{19}$, Michela Maur ${ }^{20}$, Lucia Mentuccia ${ }^{21}$, Nicla La Verde ${ }^{22}$, Giulia Bianchi23, Salvatore Artale ${ }^{24}$, Livio Blasi ${ }^{25}$, Matilde Piezzo ${ }^{26}$, Francesco Atzori $^{27}$, Anna Turletti ${ }^{28}$, Chiara Benedetto ${ }^{29}$, Maria Concetta Cursano ${ }^{30}$, Alessandra Fabi $^{31}$, Vittorio Gebbia ${ }^{32}$, Alessio Schirone ${ }^{33}$, Raffaella Palumbo ${ }^{34}$, Antonella Ferzi ${ }^{35}$, Antonio Frassoldati ${ }^{36}$, Claudio Scavellii, ${ }^{37}$ Luca Clivio ${ }^{38}$ and Valter Torri on behalf of The EVA Study Group ${ }^{38}$

${ }^{1}$ Research Unit Phase I trials, ASST Monza, Monza, Italy

2 Oncology Unit, ASST Monza, Monza, Italy

${ }^{3}$ Oncology Unit, ASST della Valle Olona-Presidio Ospedaliero di Saronno, Saronno, Italy

${ }^{4}$ Oncology Unit, Ospedale "Vito Fazzi" di Lecce, Lecce, Italy

${ }^{5}$ Oncology Unit, Ospedale Moriggia Pelascini, Gravedona, Italy

${ }^{6}$ Oncology Unit, AULSS 3, Mirano, Italy

7 Oncology Department, Policlinico di Palermo Paolo Giaccone, Palermo, Italy

${ }^{8}$ Oncology Unit 2-Città della Salute e della Scienza di Torino, Torino, Italy

${ }^{9}$ Oncology Unit, IRCCS Arcispedale S. Maria Nuova, Reggio Emilia, Italy

${ }^{10}$ Dipartimento di Scienze Cliniche Applicate e Biotecnologiche (DISCAB)-Università Degli Studi Dell'Aquila, L'Aquila, Italy

11 Oncology Unit Rimini Azienda USL Romagna, Rimini, Italy

12 Oncology Unit 2, Azienda Osp edaliera Universitaria Pisa via Roma 67, Pisa, Italy

13 Oncology Unit, ASST Papa Giovanni XXIII, Bergamo, Italy

14 Oncology Unit, Ospedale Sacro Cuore di Gesù, Fatebenefratelli, Benevento, Italy

${ }^{15}$ Brest Unit, ASST di Cremona, Cremona, Italy

${ }^{16}$ Oncology Unit, AOU Ospedali Riuniti Umberto I-GM Lancisi-G Salesi, Ancona, Italy

17 Oncology Unit, AOS Croce e Carle Ospedale di Insegnamento, Cuneo, Italy

18 Oncology Unit, Azienda Ospedaliero-Universitaria di Parma, Parma, Italy

19 Oncology Unit 2, Istituto Nazionale Tumori Regina Elena-IFO, Roma, Italy

${ }^{20}$ Oncology Unit, Policlinico University Hospital of Modena, Modena, Italy

21 Oncology Unit, ASL di Frosinone Osp. "SS. Trinità", Sora, Italy

22 Oncology Unit, ASST Fatebenefratelli Sacco Presidio Ospedaliero Fatebenefratelli, Milano, Italy

${ }^{23}$ Oncology Unit 1, Fondazione IRCCS Istituto Nazionale Tumori, Milano, Italy

24 Oncology Departement, Ospedale di Gallarate ASST Valle Olona, Gallarate, Italy

25 Oncology Unit, ARNAS Civico Palermo, Palermo, Italy

${ }^{26}$ National Cancer Institute "Fondazione Giovanni Pascale", Napoli, Italy

27 Struttura Complessa di Oncologia Medica Azienda Ospedaliero-Universitaria Cagliari, Italy

28 Oncology Unit, Ospedale Martini della ASL Città di Torino, Torino, Italy

29 Dipartimento Universitario Ginecologia e Ostetricia 1, Ospedale S. Anna Torino, Turin, Italy

30 Oncology Unit, Università Campus Bio-Medico, Roma, Italy

${ }^{31}$ Oncology Unit 1, Istituto Regina Elena-IFO, Roma, Italy 
32 Oncology Unit, Ospedale La Maddalena, Palermo, Italy

33 Oncolgy Department, Istituto Scientifico Romagnolo per lo Studio e la Cura dei Tumori (IRST) IRCCS, Meldola, Italy

34 Oncology Unit, IRCCS ICS Maugeri, Pavia, Italy

35 Oncology Unit, ASST OVEST Milanese, Presidio di Legnano, Legnano, Italy

36 Oncology Unit, Az Ospedaliero Universitaria di Ferrara, Ferrara, Italy

37 Oncology Unit, Ospedale "S. Cuore di Gesù", Gallipoli, Italy

38 IRCCS-Istituto di Ricerche Farmacologiche "Mario Negri", Milano, Italy

Published: October 02, 2018

Copyright: Cazzaniga et al. This is an open-access article distributed under the terms of the Creative Commons Attribution License 3.0 (CC BY 3.0), which permits unrestricted use, distribution, and reproduction in any medium, provided the original author and source are credited.

This article has been corrected: The correct author list and Appendix 1 are given below:

Marina Cazzaniga ${ }^{1,2}$, Claudio Verusio ${ }^{3}$, Mariangela Ciccarese ${ }^{4}$, Alberto Fumagalli ${ }^{5}$, Donata Sartori ${ }^{6}$, Maria Rosaria Valerio', Mario Airoldi ${ }^{8}$, Gabriella Moretti ${ }^{9}$, Corrado Ficorella ${ }^{10}$, Valentina Arcangelii ${ }^{11}$, Lucrezia Diodati ${ }^{12}$, Alberto Zambelli ${ }^{13}$, Antonio Febbraro $^{14}$, Daniele Generali ${ }^{15}$, Mirco Pistelli' ${ }^{16}$, Ornella Garrone ${ }^{17}$, Antonino Musolino ${ }^{18}$, Patrizia Vici ${ }^{19}$, Michela Maur ${ }^{20}$, Lucia Mentuccia ${ }^{21}$, Nicla La Verde ${ }^{22}$, Giulia Bianchi ${ }^{23}$, Salvatore Artale ${ }^{24}$, Livio Blasi ${ }^{25}$, Matilde Piezzo ${ }^{26}$, Francesco Atzori $^{27}$, Anna Turletti ${ }^{28}$, Chiara Benedetto ${ }^{29}$, Maria Concetta Cursano ${ }^{30}$, Alessandra Fabi $^{31}$, Vittorio Gebbia ${ }^{32}$, Alessio Schirone ${ }^{33}$, Raffaella Palumbo ${ }^{34}$, Antonella Ferzi ${ }^{35}$, Antonio Frassoldati ${ }^{36}$, Claudio Scavelli ${ }^{37}$, Luca Clivio ${ }^{38}$ and Valter Torri on behalf of The EVA Study Group ${ }^{38}$

\section{Appendix 1}

List of Co-Authors

Cicchiello F. ${ }^{2}$, Riva F. ${ }^{2}$, Ballerio A. ${ }^{3}$, Coccato S. ${ }^{6}$, Ancona C. ${ }^{7}$, De Martini P. ${ }^{8}$, Cocciolone V. ${ }^{10}$, Gianni L. ${ }^{11}$, Michelotti A. ${ }^{12}$, Campidoglio S..$^{14}$, Dester M..$^{15}$, Pizzuti L. ${ }^{1}$, Caggia F. ${ }^{20}$, Ferrari L. ${ }^{21}$, Girelli S. ${ }^{22}$, Bedolis R. ${ }^{24}$, Alù M..$^{25}$, De Laurentiis M. ${ }^{26}$, Bordin E. ${ }^{28}$, Porpiglia M. ${ }^{29}$ Santini D. ${ }^{30}$, Bozzoli E. ${ }^{31}$, Marchese A. ${ }^{32}$.

${ }^{2}$ Oncology Unit, ASST Monza, Monza, Italy.

${ }^{3}$ Oncology Unit, ASST della Valle Olona - Presidio Ospedaliero di Saronno, Saronno, Italy.

${ }^{6}$ Oncology Unit, AULSS 3 Mirano, Italy.

7 Oncology Department, Policlinico di Palermo Paolo Giaccone Palermo, Italy.

${ }^{8}$ Oncology Unit 2 - Città della Salute e della Scienza di Torino, Torino, Italy.

${ }^{10}$ Dipartimento di Scienze Cliniche Applicate e Biotecnologiche (DISCAB) - Università Degli Studi Dell'Aquila.

${ }^{11}$ Oncology Unit Rimini Azienda USL Romagna, Rimini, Italy.

12 Oncology Unit 2, Azienda Ospedaliera Universitaria Pisa via Roma 67 Pisa, Italy.

${ }^{14}$ Oncology Unit, Ospedale Sacro Cuore di Gesù, Fatebenefratelli, Benevento, Italy.
${ }^{15}$ Breast Unit, ASST di Cremona, Italy.

19 Oncology Unit 2, Istituto Nazionale Tumori Regina Elena - IFO, Roma, Italy.

${ }^{20}$ Oncology Unit, Policlinico University Hospital of Modena, Italy.

${ }^{21}$ Oncology Unit, ASL di Frosinone Osp. "SS. Trinità", Italy.

${ }^{22}$ Oncology Unit, ASST Fatebenefratelli Sacco Presidio Ospedaliero Fatebenefratelli, Milano, Italy.

${ }^{24}$ Oncology Departement, Ospedale di Gallarate ASST Valle Olona, Gallarate, Italy.

25 Oncology Unit, ARNAS Civico Palermo, Palermo, Italy.

${ }^{26}$ National Cancer Institute "Fondazione Giovanni Pascale", Napoli, Italy.

${ }^{28}$ Ospedale Martini della ASL "Città di Torino.

29 Dipartimento Universitario Ginecologia e Ostetricia 1, Ospedale S. Anna Torino, Italy.

${ }^{30}$ Oncology Unit, Università Campus Bio-Medico, Roma, Italy.

${ }^{31}$ Oncology Unit 1, Istituto Regina Elena - IFO, Roma, Italy.

${ }^{32}$ Oncology Unit, Ospedale Sacro Cuore di Gesù, Fatebenefratelli, Benevento, Italy.

Original article: Oncotarget. 2018; 9:31877-31887. https://doi.org/10.18632/oncotarget.25874 